\title{
ODD STRUCTURES ARE ODD
}

\author{
MARTIN MARKL
}

\begin{abstract}
By an odd structure we mean an algebraic structure in the category of graded vector spaces whose structure operations have odd degrees. Particularly important are odd modular operads which appear as Feynman transforms of modular operads and, as such, describe some structures of string field theory.

We will explain how odd structures are affected by the choice of the monoidal structure of the underlying category. We will then present two 'natural' and 'canonical' constructions of an odd modular endomorphism operad leading to different results, only one being correct. This contradicts the generally accepted belief that the systematic use of the Koszul sign rule leads to correct signs.
\end{abstract}

\section{CONTENTS}

Introduction

1. The category of graded vector spaces

2. Odd modular operads 5

3. Odd endomorphism operads 8

References

\section{INTRODUCTION}

As noticed in the seminal paper [2], the category of modular operads is not Koszul selfdual. Consequently, the bar construction of a modular operad (called in this context the Feynman transform) is not an ordinary modular operad, but an odd modular operad. It was shown in [4] and in the forthcoming work [1, 6, 7] that some algebraic structures relevant for string field theory are algebras over the Feynman transform of a modular operad. This explains the interest in explicit understanding odd modular operads and their algebras.

Odd modular operads live in the category Vect of graded vector spaces and their linear homogeneous maps of arbitrary degrees. This category is enriched over the category Vect of graded vector spaces and linear maps of degree 0 and admits two different yet 'natural' and 'canonical' symmetric closed monoidal structures.

Structural operations of odd modular operads have degrees +1 , so they are examples of an 'odd' structure having operations of odd degrees. We use odd associative algebras as a simple example which shows that concrete 'models' of such structures might depend on the choice

2010 Mathematics Subject Classification. 18D50 (Primary), 18D20, 18D10 (Secondary).

Key words and phrases. Graded vector space, monoidal structure, odd endomorphism operad.

The author was supported by the Eduard Čech Institute P201/12/G028 and RVO: 67985840.

${ }^{\dagger}$ Terminology suggested by Ralph Kaufmann; a definition is recalled in Section 3 . 
of a monoidal structure of Vect. The same is very crucially true also for odd modular operads, the main subject of this note. The 'oddness' of operad- and PROP-like structures was discussed in great detail in [3]; we refer to it for other examples of odd structures.

The concept of algebras over odd modular operads requires odd endomorphism operads; and algebra over an odd modular operad $\mathfrak{l}_{\mathfrak{}}$ is a morphism $\mathfrak{} \rightarrow \mathcal{E} n d_{V}$ from $\mathfrak{l}_{\text {to }}$ the odd endomorphism operad $\mathcal{E} n d_{V}$. The structure of the odd endomorphism operad is extremely sign-sensitive. We will present two 'natural' and 'canonical' constructions of this operad, leading quite unexpectedly to different results. This phenomenon is explained by the presence of two different monoidal structures of Vect. Proposition 6 of the last section specifies which one gives the correct result.

The moral is that even the systematic and careful use of the Koszul sign rule might lead to wrong results if one is unlucky. We hope that this note would warn the reader that this may indeed happen if 'odd' structures are present.

Conventions. All algebraic objects will be considered over a fixed field $\mathrm{k}$ of characteristic zero. The symbol $\otimes$ will be reserved for the tensor product over $k$. We will denote by $1_{X}$ or simply by 1 when $X$ is understood, the identity endomorphism of an object $X$ (set, vector space, \&c.).

By a grading we mean a Z-grading, though everything in this note can easily be modified to the $Z_{2}$-graded case. The degree of a graded object will be denoted by $|w|$. We will use the Koszul sign rule meaning that whenever we commute two "things" of degrees $p$ and $q$, respectively, we multiply the sign by $(-1)^{p q}$.

We assume basic knowledge of operads with the emphasis on modular ones as it can be gained for example from [5, Chapter 5] complemented by the original source [2].

Notation. For $n \geq 1$ we denote by $\Sigma_{n}$ the symmetric group of $n$ elements realized as the group of automorphism of the set $\{1, \ldots, n\}$. For graded indeterminates $x_{1}, \ldots, x_{n}$ and a permutation $\sigma \in \Sigma_{n}$ we define the Koszul sign $\epsilon(\sigma)=\epsilon\left(\sigma ; x_{1}, \ldots, x_{n}\right)$ by

$$
x_{1} \cdots x_{n}=\epsilon\left(\sigma ; x_{1}, \ldots, x_{n}\right) \cdot x_{\sigma(1)} \cdots x_{\sigma(n)},
$$

which has to be satisfied in the free graded commutative associative algebra $\mathrm{S}\left(x_{1}, \ldots, x_{n}\right)$ generated by $x_{1}, \ldots, x_{n}$.

For graded vector spaces $V$ and $W$ we denote by $\operatorname{Vect}^{k}(V, W)$ the vector space of degree $k$ morphisms $V \rightarrow W$ and by $\underline{\operatorname{Vect}}(V, W)$ the graded vector space

$$
\underline{\operatorname{Vect}}(V, W):=\bigoplus_{k \in \mathrm{Z}} \underline{\operatorname{Vect}}^{k}(V, W) .
$$

If $W$ is the ground field $\mathrm{k}$, we obtain the graded dual $V^{*}:=\operatorname{Vect}(V, \mathrm{k})$ of $V$. Notice that the degree $k$ component of $V^{*}$ equals the standard linear dual $\left(V^{-k}\right)^{*}$ of the degree $-k$ component of $V$. A degree $k$ morphism $f: V \rightarrow W$ defines a map $f^{*}: W^{*} \rightarrow V^{*}$ of the same degree by the formula

$$
f^{*}(x):=(-1)^{k|x|} x \circ f, x \in V^{*} .
$$

For $V=\bigoplus_{p} V_{p}$, let $\uparrow V$ be the suspension of $V$, i.e. the graded vector space defined by $(\uparrow V)_{p}=V_{p-1}$. One has the obvious linear isomorphisms $\uparrow: V \rightarrow \uparrow V$ and $\downarrow: \uparrow V \rightarrow V$ of degrees +1 and -1 , respectively. Given graded vector spaces $V_{1}$ and $V_{2}$, we denote by $\tau$ the

[August 10, 2016]

[odd.tex] 
symmetry

$$
\tau: V_{1} \otimes V_{2} \rightarrow V_{1} \otimes V_{2}, \tau\left(v_{1} \otimes v_{2}\right):=(-1)^{\left|v_{1}\right|\left|v_{2}\right|}\left(v_{2} \otimes v_{1}\right)
$$

Acknowledgment. Main ideas of this note were born during conversations with Michael Batanin and Martin Doubek. Martin pointed to me the potential sign problems in the construction of the odd endomorphism operad, and Michael substantially contributed to my understanding of the categories of graded vector spaces. I owe my thanks to Bruno Vallette for explaining to me the difference between the Koszul sign convention and the Koszul sign rule, and to Ralph Kaufmann for turning my attention to [3]. I enjoyed the wonderful atmosphere of the Max-Planck Institut für Matematik in Bonn in the period when this paper was completed. I am also indebted to an anonymous referee for many useful remarks and corrections.

Plan of the paper. In Section 11 we show that the 2-category Cat(Vect) of categories enriched over the category Vect of graded vector spaces and their linear degree 0-maps admits two monoidal structures, $\odot_{\mathrm{S}}$ and $\odot_{\mathrm{M}}$, thus one has two types of pseudomonoids in Cat(Vect). We notice that the category Vect of graded vector spaces and their linear maps of arbitrary degrees with its standard Vect-enriched monoidal (tensor) structure is a pseudomonoid for $\odot_{\mathrm{S}}$, while it has yet another monoidal structure which makes it a pseudomonoid for $\odot_{\mathrm{M}}$. Section 1 is complemented with a toy example of an odd structure in Vect.

In Section 2 we recall odd modular operads and show how their concrete models depend on the choice of a monoidal structure of Vect. The last section is devoted to two constructions of the odd endomorphism operad. We investigate the sensitivity of these constructions to the monoidal structure of $\underline{\text { Vect. }}$.

\section{The CATEGory of GRADED VECTOR SPACES}

Let Vect denote the category of graded vector spaces and their linear degree 0-maps, and Cat(Vect) the 2-category of Vect-enriched categories. The 2-category Cat(Vect) bears the 'standard' monoidal structure, denoted $\odot_{\mathrm{S}}$, defined as follows. For enriched categories $\mathrm{A}, \mathrm{B} \in \mathrm{Cat}$ (Vect), the objects of the category $\mathrm{A} \odot_{\mathrm{S}} \mathrm{B}$ are couples $(a, b)$, where $a$ is an object of $\mathrm{A}$ and $b$ an object of $\mathrm{B}$. The enriched hom-spaces are

$$
\left(\mathrm{A} \odot_{\mathrm{S}} \mathrm{B}\right)\left(\left(a_{1}, b_{1}\right),\left(a_{2}, b_{2}\right)\right):=\mathrm{A}\left(a_{1}, a_{2}\right) \otimes \mathrm{B}\left(b_{1}, b_{2}\right),
$$

and the enriched composition ${ }^{\circ}$ is given by the diagram

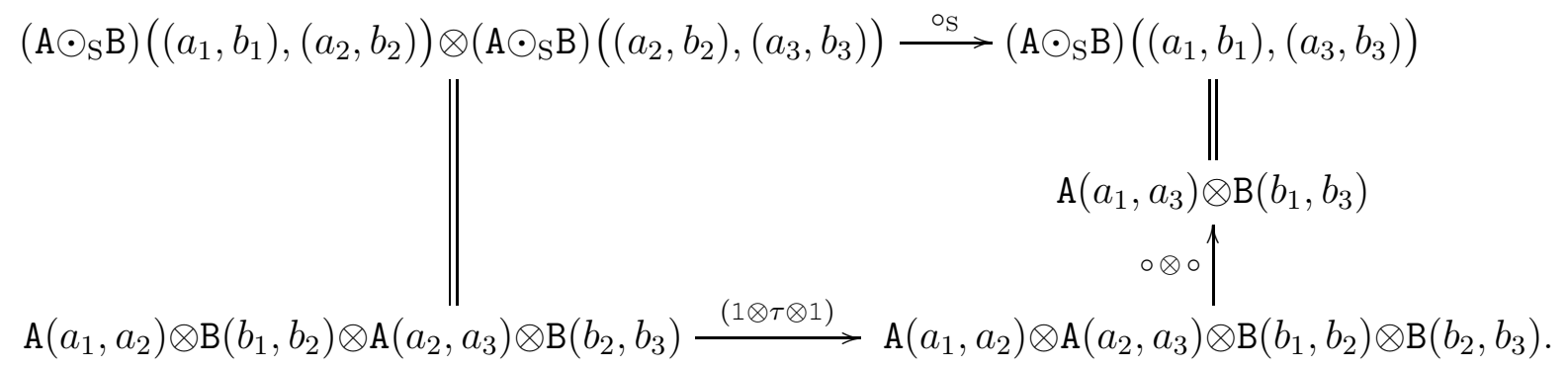

In the above displays, $a_{1}, a_{2}, a_{3}$ are objects of $\mathrm{A}, b_{1}, b_{2}, b_{3}$ objects of $\mathrm{B}, \tau$ the symmetry (3) and $\circ$ the compositions in A resp. B. The $\circ_{S}$-composition can also be defined directly by [odd.tex]

[August 10, 2016] 
the formula

$$
\left(f_{1} \otimes g_{1}\right) \circ_{\mathrm{S}}\left(f_{2} \otimes g_{2}\right):=(-1)^{\left|g_{1}\right|\left|f_{2}\right|}\left(f_{1} \circ f_{2} \otimes g_{1} \circ g_{2}\right),
$$

where $f_{1}, f_{2}$ are composable morphisms of $\mathrm{A}$ and $g_{1}, g_{2}$ composable morphisms of B.

The 2-category Cat(Vect) has however another monoidal structure which we denote by $\odot_{\mathrm{M}}$ and call it, from the reasons explained later, the $M c$ Gill monoidal structure. The objects of $\mathrm{A} \odot_{\mathrm{M}} \mathrm{B}$ are the same as the objects of $\mathrm{A} \odot_{\mathrm{S}} \mathrm{B}$, and also the enriched hom-spaces agree, i.e.

$$
\left(\mathrm{A} \odot{ }_{\mathrm{M}} \mathrm{B}\right)\left(\left(a_{1}, b_{1}\right),\left(a_{2}, b_{2}\right)\right):=\mathrm{A}\left(a_{1}, a_{2}\right) \otimes \mathrm{B}\left(b_{1}, b_{2}\right)
$$

but the composition $\circ_{\mathrm{M}}$ is now given by

$$
\left(f_{1} \otimes g_{1}\right) \circ_{\mathrm{M}}\left(f_{2} \otimes g_{2}\right):=(-1)^{\left|f_{1}\right|\left|g_{2}\right|}\left(f_{1} \circ f_{2} \otimes g_{1} \circ g_{2}\right)
$$

The monoidal 2-categories $\left(\operatorname{Cat}(\right.$ Vect $\left.), \odot_{\mathrm{S}}\right)$ and $\left(\operatorname{Cat}(\right.$ Vect $\left.), \odot_{\mathrm{M}}\right)$ are isomorphic as monoidal categories, via the isomorphism $\pi$ given by the identity on Cat(Vect) and the natural family of functors

$$
\left\{\varpi_{\mathrm{A}, \mathrm{B}}: \mathrm{A} \odot_{\mathrm{S}} \mathrm{B} \stackrel{\cong}{\longrightarrow} \mathrm{A} \odot_{\mathrm{M}} \mathrm{B}, \mathrm{A}, \mathrm{B} \in \operatorname{Cat}(\text { Vect })\right\} .
$$

The functor $\varpi_{A, B}$ is the identity on objects, while on a morphism

$$
f \otimes g \in \mathrm{A}\left(a_{1}, a_{2}\right) \otimes \mathrm{B}\left(b_{1}, b_{2}\right)=\left(\mathrm{A} \odot_{\mathrm{S}} \mathrm{B}\right)\left(\left(a_{1}, b_{1}\right),\left(a_{2}, b_{2}\right)\right)
$$

it acts as

$$
\varpi_{\mathrm{A}, \mathrm{B}}(f \otimes g):=(-1)^{|f||g|}(f \otimes g) \in \mathrm{A}\left(a_{1}, a_{2}\right) \otimes \mathrm{B}\left(b_{1}, b_{2}\right)=\left(\mathrm{A} \odot_{\mathrm{M}} \mathrm{B}\right)\left(\left(a_{1}, b_{1}\right),\left(a_{2}, b_{2}\right)\right) .
$$

The category Vect of graded vector spaces and their homogeneous linear maps of arbitrary degrees is naturally enriched over Vect. It turns out that also Vect admits two Vectenriched symmetric monoidal structures, the standard one and the McGill monoidal structure defined below.

The monoidal product of objects is for both structures the usual tensor product of graded vector spaces, but the products differ by their actions on morphisms. The standard convention is that, for homogeneous maps $f: V^{\prime} \rightarrow W^{\prime}, g: V^{\prime \prime} \rightarrow W^{\prime \prime}$ and homogeneous vectors $u \in V^{\prime}, v \in W^{\prime}$ one defines

$$
(f \otimes g)(u \otimes v)=(-1)^{|g||u|} f(u) \otimes g(v),
$$

while some categorists at McGill University in Montreal would prefer

$$
(f \otimes g)(u \otimes v)=(-1)^{|f||v|} f(u) \otimes g(v) .
$$

The second convention would follow from the Koszul sign rule if we are applying the morphisms from the right. Equation (4b) would then read as

$$
(u \otimes v)(f \otimes g)=(-1)^{|f||v|} f(u) \otimes g(v),
$$

the unexpected sign coming from commuting $f$ over $v$. We denote, only for the purposes of this section, the first monoidal structure by $\otimes_{\mathrm{S}}$ and second by $\otimes_{\mathrm{M}}$ (' $\mathrm{S}$ ' abbreviating

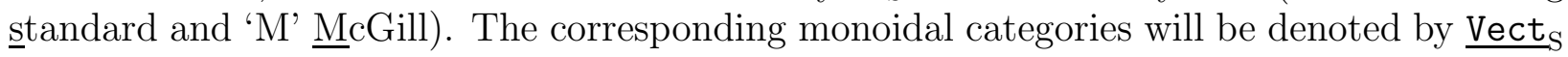
and $\underline{\mathrm{Vect}}_{\mathrm{M}}$, respectively. Notice that both monoidal structures coincide on the subcategory Vect of graded vector spaces and their linear degree 0 maps.

It can be easily verified that $\underline{\text { Vect }}_{S}$ is a symmetric pseudomonoid in (Cat (Vect), $\odot_{S}$ ) while $\underline{\text { Vect }}_{M}$ a symmetric pseudomonoid in (Cat $($ Vect $\left.), \odot_{\mathrm{M}}\right)$. The isomorphism

$$
\pi:\left(\operatorname{Cat}(\operatorname{Vect}), \odot_{\mathrm{S}}\right) \cong\left(\operatorname{Cat}(\operatorname{Vect}), \odot_{\mathrm{M}}\right)
$$

[August 10, 2016]

[odd.tex] 
induces an isomorphism

$$
\operatorname{PsMon}(\pi): \operatorname{PsMon}\left(\operatorname{Cat}(\text { Vect }), \odot_{\mathrm{S}}\right) \cong \operatorname{PsMon}\left(\operatorname{Cat}(\text { Vect }), \odot_{\mathrm{M}}\right)
$$

of the corresponding categories of pseudomonoids, and

$$
\operatorname{PsMon}(\pi)\left(\underline{\operatorname{Vect}}_{\mathrm{S}}\right)=\underline{\operatorname{Vect}}_{\mathrm{M}} .
$$

We finish this section by a kindergarten example of an odd structure in Vect. An odd associative algebra is a couple $A=(A, \bullet)$ consisting of a graded vector space $A$ and a degree +1 operation $\bullet: A \otimes A \rightarrow A$ which is anti-associative, i.e.

$$
\bullet(1 \otimes \bullet)+\bullet(\bullet \otimes 1)=0 \text {. }
$$

Since the structure operation has an odd degree, the form of axiom (5) evaluated at concrete elements may depend on the chosen monoidal structure of Vect. Let us calculate

$$
\bullet(1 \otimes \bullet)(x \otimes y \otimes z)+\bullet(\bullet \otimes 1)(x \otimes y \otimes z) .
$$

for all $x, y, z \in A$. While in $\underline{\text { Vect }}_{\mathrm{S}}$ we get

$$
(-1)^{|x|} x \bullet(y \bullet z)+(x \bullet y) \bullet z,
$$

in $\underline{\text { Vect }}_{M}$ we obtain

$$
x \bullet(y \bullet z)+(-1)^{|z|}(x \bullet y) \bullet z .
$$

The categories of odd associative algebras in $\underline{\text { Vect }}_{S}$ and in $\underline{\text { Vect }}_{M}$ are however isomorphic. Indeed, the modification

$$
x \bullet y \longmapsto(-1)^{|x|+|y|} x \bullet y
$$

turns an odd associative algebra in $\underline{\text { Vect }}_{S}$ into one in $\underline{\text { Vect }}_{\mathrm{M}}$ and vice versa. In fact, there exists an one-to-one correspondence between odd associative algebra structures on $A$ and usual associative algebra structures on the suspension $\uparrow A$. The corresponding associative product $\circ$ is given by the commutative diagram

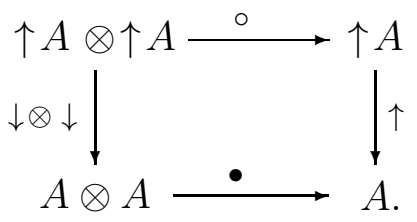

If not stated otherwise, we will use in the rest of this note the standard monoidal structure and drop the subscript $\mathrm{S}$.

\section{ODD MODULAR OPERADS}

Odd modular operads are particular cases of twisted modular operads which were introduced in the classical 1998 paper [2, (4.2)]. They were originally defined as algebras for a certain monad of decorated graphs. For practical calculations it is however convenient to have a biased definition which appeared much later in [1]. For the convenience of the reader we repeat it below. The first definition in which Vect denotes the category of graded vector space and their degree-0 linear maps is however standard.

\footnotetext{
${ }^{\ddagger}$ More precisely, they are $\mathfrak{K}$-twisted operads, where $\mathfrak{K}$ is the dualizing cocycle [2, (4.8)]. [odd.tex]

[August 10, 2016]
} 
Definition 1. A modular module (in Vect) is a covariant functor

$$
E: \mathrm{fSet} \times \mathrm{N} \rightarrow \text { Vect }
$$

from the cartesian product of the category fSet of finite sets and their isomorphisms with the discrete category of natural numbers to Vect.

Explicitly, a modular module $E$ is a collection $E(S ; g), S \in \mathrm{fSet}, g \in \mathrm{N}$, of graded vector spaces together with functorial degree 0 morphisms

$$
E(\sigma): E(S ; g) \rightarrow E(T ; g)
$$

specified for any isomorphism $\sigma: S \stackrel{\text { ? }}{\longrightarrow} T$ and $g \in \mathrm{N}$.

Roughly speaking, an odd modular operad is a modular operad whose structure operations have 'wrong' degrees and some axioms acquire 'wrong' signs. Let us give a precise:

Definition 2. An odd modular operad is a modular module

$$
\boldsymbol{\Omega}=\{\boldsymbol{Q}(S ; g) \in \operatorname{Vect} \mid(S ; g) \in \mathrm{fSet} \times \mathrm{N}\}
$$

together with degree +1 morphisms $\left({ }_{a} \bullet_{b}\right.$-operations $)$

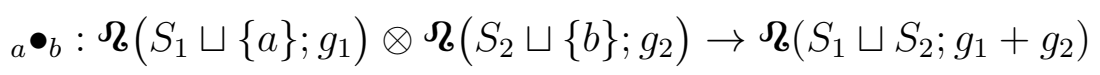

defined for arbitrary disjoint finite sets $S_{1}, S_{2}$, symbols $a, b$, and arbitrary $g_{1}, g_{2} \in \mathrm{N}$. There are, moreover, degree 1 morphisms (the contractions)

$$
\bullet_{u v}=\bullet_{v u}: \boldsymbol{\Omega}(S \sqcup\{u, v\} ; g) \rightarrow \boldsymbol{\Omega}(S ; g+1)
$$

given for any finite set $S, g \in \mathrm{N}$, and symbols $u, v$. These data are required to satisfy the following axioms.

(i) For arbitrary isomorphisms $\rho: S_{1} \sqcup\{a\} \rightarrow T_{1}$ and $\sigma: S_{2} \sqcup\{b\} \rightarrow T_{2}$ of finite sets and $g_{1}, g_{2} \in \mathrm{N}$, one has the equality

$$
\boldsymbol{\mathcal { }}\left(\left.\left.\rho\right|_{S_{1}} \sqcup \sigma\right|_{S_{2}}\right)_{a} \bullet_{b}={ }_{\rho(a)} \bullet_{\sigma(b)}(\boldsymbol{\mathcal { Q }}(\rho) \otimes \boldsymbol{\mathcal { }}(\sigma))
$$

of maps

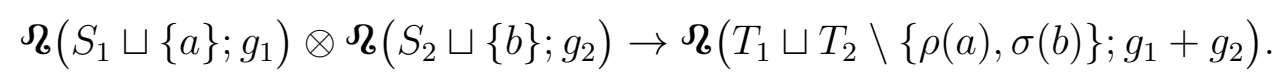

(ii) For an isomorphism $\rho: S \sqcup\{u, v\} \rightarrow T$ of finite sets and $g \in \mathrm{N}$, one has the equality

$$
\boldsymbol{\mathcal { Z }}\left(\left.\rho\right|_{S}\right) \bullet \boldsymbol{\bullet}_{u v}=\bullet \rho(u) \rho(v) \boldsymbol{\mathcal { }}(\rho)
$$

of maps $\boldsymbol{\Omega}(S \sqcup\{u, v\} ; g) \rightarrow \boldsymbol{\Omega}(T \backslash\{\rho(u), \rho(v)\} ; g+1)$.

(iii) For $S_{1}, S_{2}, a, b$ and $g_{1}, g_{2}$ as in (6), one has the equality

$$
\begin{gathered}
\boldsymbol{a}_{b}={ }_{b} \bullet_{a} \tau \\
\text { of maps } \boldsymbol{\Omega}\left(S_{1} \sqcup\{a\} ; g_{1}\right) \otimes \boldsymbol{\Omega}\left(S_{2} \sqcup\{b\} ; g_{2}\right) \rightarrow \boldsymbol{\Omega}\left(S_{1} \sqcup S_{2} ; g_{1}+g_{2}\right) \text {. }
\end{gathered}
$$

\footnotetext{
${ }^{\S}$ Isomorphisms are the only morphisms in $\mathrm{f}$ Set by definition.

"We are using the notation for structure operations of odd modular operads introduced in [3].

"Recall that $\tau$ is the commutativity constraint (3) in the category of graded vector spaces.
}

[August 10, 2016]

[odd.tex] 
(iv) For mutually disjoint sets $S_{1}, S_{2}, S_{3}$, symbols $a, b, c, d$ and $g_{1}, g_{2}, g_{3} \in \mathrm{N}$, one has the equality

$$
{ }_{a} \bullet_{b}\left(1 \otimes{ }_{c} \bullet_{d}\right)=-{ }_{c} \bullet_{d}\left({ }_{a} \bullet_{b} \otimes 1\right)
$$

of maps from $\boldsymbol{\Omega}\left(S_{1} \sqcup\{a\} ; g_{1}\right) \otimes \boldsymbol{\Omega}\left(S_{2} \sqcup\{b, c\} ; g_{2}\right) \otimes \boldsymbol{\Omega}\left(S_{3} \sqcup\{d\} ; g_{3}\right)$ to the space $\boldsymbol{\Omega}\left(S_{1} \sqcup S_{2} \sqcup S_{3} ; g_{1}+g_{2}+g_{3}\right)$.

(v) For a finite set $S$, symbols $a, b, c, d$ and $g \in \mathrm{N}$ one has the equality

$$
\bullet_{a b} \bullet_{c d}=-\bullet_{c d} \bullet_{a b}
$$

of maps $\boldsymbol{\Omega}(S \sqcup\{a, b, c, d\} ; g) \rightarrow \boldsymbol{\Omega}(S ; g+2)$.

(vi) For finite sets $S_{1}, S_{2}$, symbols $a, b, c, d$ and $g_{1}, g_{2} \in \mathrm{N}$, one has the equality

$$
\begin{gathered}
\bullet_{a b} \bullet_{d}=-\bullet_{c d} \quad{ }_{a} \bullet_{b} \\
\text { of maps } \boldsymbol{\Omega}\left(S_{1} \sqcup\{a, c\} ; g_{1}\right) \otimes \boldsymbol{\Omega}\left(S_{2} \sqcup\{b, d\} ; g_{2}\right) \rightarrow \boldsymbol{\Omega}\left(S_{1} \sqcup S_{2} ; g_{1}+g_{2}+1\right) .
\end{gathered}
$$

(vii) For finite sets $S_{1}, S_{2}$, symbols $a, b, u, v$, and $g_{1}, g_{2} \in \mathrm{N}$, one has the equality

$$
\begin{gathered}
{ }_{a} \bullet_{b}\left(\bullet_{u v} \otimes 1\right)=-\bullet_{u v} \quad{ }_{a} \bullet_{b} \\
\text { of maps } \boldsymbol{\Omega}\left(S_{1} \sqcup\{a, u, v\} ; g_{1}\right) \otimes \boldsymbol{\Omega}\left(S_{2} \sqcup\{b\} ; g_{2}\right) \rightarrow \boldsymbol{\Omega}\left(S_{1} \sqcup S_{2} ; g_{1}+g_{2}+1\right) .
\end{gathered}
$$

As for odd associative algebras discussed in Section 1, the form of some axioms of odd modular operads evaluated at concrete elements depends on the chosen monoidal structure of Vect. Let us, for instance, evaluate axiom (iv) at homogeneous elements

$$
\left.x \in \boldsymbol{\Omega}\left(S_{1} \sqcup\{a\} ; g_{1}\right), y \in \boldsymbol{\Omega}_{(} S_{2} \sqcup\{b, c\} ; g_{2}\right) \text { and } z \in \boldsymbol{\Omega}\left(S_{3} \sqcup\{d\} ; g_{3}\right)
$$

i.e. expand

$$
{ }_{a} \bullet_{b}\left(1 \otimes{ }_{c} \bullet_{d}\right)(x \otimes y \otimes z)=-{ }_{c} \bullet_{d}\left({ }_{a} \bullet_{b} \otimes 1\right)(x \otimes y \otimes z) .
$$

While in $\underline{\text { Vect }}_{S}$ we get

$$
(-1)^{|x|} x_{a} \bullet_{b}\left(y_{c} \bullet_{d} z\right)=-\left(x_{a} \bullet_{b} y\right)_{c} \bullet_{d} z,
$$

in $\underline{\text { Vect }}_{M}$ we obtain

$$
x_{a} \bullet_{b}\left(y_{c} \bullet_{d} z\right)=-(-1)^{|z|}\left(x_{a} \bullet_{b} y\right)_{c} \bullet_{d} z .
$$

Likewise, axiom (vii) in $\underline{\text { Vect }}_{\mathrm{S}}$ reads

$$
\bullet_{u v}(x)_{a} \bullet_{b} y=-\bullet_{u v}\left(x_{a} \bullet_{b} y\right)
$$

while in $\underline{\text { Vect }}_{M}$ one would get

$$
(-1)^{|y|} \bullet_{u v}(x)_{a} \bullet_{b} y=-\bullet_{u v}\left(x_{a} \bullet_{b} y\right)
$$

for $x, y$ belonging to the appropriate components of $\mathcal{\complement}$. The remaining axioms are the same in both monoidal structures.

It turns our that the categories of odd modular operads in $\underline{\text { Vect }}_{\mathrm{S}}$ and in $\underline{\text { Vect }}_{\mathrm{M}}$ are isomorphic; the modification

$$
x_{a} \bullet_{b} y \mapsto(-1)^{|x|+|y|} x_{a} \bullet \bullet_{b} y, \bullet_{u v}(x) \mapsto(-1)^{|x|} \bullet_{u v}(x),
$$

turns an odd modular operad in $\underline{\text { Vect }}_{S}$ into one in $\underline{\text { Vect }}_{M}$ and vice versa.

It is however not true that an odd modular operad structure is the same as an ordinary one on the suspension of the underlying modular module. While the suspended ${ }_{a} \bullet^{-}$-operations are of degree 0 as for the ordinary modular operads, the suspended contractions $\bullet_{u v}$ retain degree 1. The categories of ordinary and odd modular operads are genuinely different. [odd.tex]

[August 10, 2016] 


\section{ODD ENDOMORPHISM OPERADS}

The classical definition of the (ordinary) modular endomorphism operad $\mathcal{E}_{n} d_{V}$ given in [2, (1.7)] requires as the input data a graded vector space $V$ with a non-degenerate symmetric bilinear form $B: V \otimes V \rightarrow \mathrm{k}$ of degree 0 . For $[n]:=\{1, \ldots, n\}$ one puts

$$
\mathcal{E}_{n} d_{V}([n]):=V^{\otimes n}, n \geq 0
$$

with the operadic structure given by 'contracting indexes' using $B$. If $B$ has degree +1 , the same construction leads to an odd modular endomorphism operad [4, Example 5.3].

One easily observes that $B$ need not be non-degenerate - everything makes sense even in the extreme case when $B=0$. Moreover, in mathematical physics, it is more natural to work in the dual settig with a symmetric degree +1 tensor $s \in V \otimes V$ instead of $B$, and the components of the odd endomorphism operad given by

$$
\mathcal{E} n d_{V}([n]):=\left(V^{\otimes n}\right)^{*}, n \geq 0 .
$$

The operadic structure is given by 'expanding indexes' using $s$. We will focus to this version of the odd endomorphism operad.

It turns out that there are two interpretations what expanding indexes means. The first, seemingly preferable one, is expressed by (12a) and (13a) below. It does not involve duals and uses only canonical isomorphisms. The other one, represented by (12b) and (13b), is much less aesthetically pleasing since it needs duals and inclusions of the form

$$
A^{*} \otimes B^{*} \hookrightarrow(A \otimes B)^{*}
$$

which from seemingly random reasons go in the desired direction. It is the peculiarity of the odd case that both constructions lead to different results. If we assume the standard monoidal structure of Vect, then the correct result is given by the second, ugly one.

We will need the tensor product of a family $\left\{V_{c}\right\}_{c \in S}$ of graded vector spaces indexed by a finite set $S \in$ fSet. Since $S$ is not a priory ordered, we want a concept that would not depend on a chosen order. The idea is to choose an order, then perform the usual tensor product, and then identify the products over different orders using the Koszul sign rule. Since an order of a finite set $S$ with $n$ elements is the same as an isomorphism $\omega:\{1, \ldots, n\} \stackrel{\cong}{\rightarrow} S$, we are led to the following:

Definition 3. The unordered tensor product $\bigotimes_{c \in S} V_{c}$ of the collection $\left\{V_{c}\right\}_{c \in S}$ is the vector space of equivalence classes of usual tensor products

$$
v_{\omega(1)} \otimes \cdots \otimes v_{\omega(n)} \in V_{\omega(1)} \otimes \cdots \otimes V_{\omega(n)}, \omega:\{1, \ldots, n\} \stackrel{\cong}{\longrightarrow} S
$$

modulo the identifications

$$
v_{\omega(1)} \otimes \cdots \otimes v_{\omega(n)} \sim \epsilon(\sigma) v_{\omega \sigma(1)} \otimes \cdots \otimes v_{\omega \sigma(n)}, \sigma \in \Sigma_{n}
$$

where $\epsilon(\sigma)$ is the Koszul sign (1) of the permutation $\sigma$.

The need for a subtler version of the tensor product is caused by the fact that the category Vect of graded vector spaces is a symmetric monoidal category with a non-trivial symmetry. Similar unordered products can be defined in any symmetric monoidal category with finite colimits, see e.g. [5, Def. II.1.58]. Let us formulate two important properties of unordered tensor products.

[August 10, 2016]

[odd.tex] 
Lemma 4. Let $\sigma: S \rightarrow D$ be an isomorphism of finite sets, $\left\{V_{c}\right\}_{c \in S}$ and $\left\{W_{d}\right\}_{d \in D}$ collections of graded vector spaces, and $\varphi=\left\{\varphi_{c}: V_{c} \rightarrow W_{\sigma c}\right\}_{c \in S}$ a family of linear maps. Then the assignment

$$
\bigotimes_{c \in S} V_{c} \ni\left[v_{\omega(1)} \otimes \cdots \otimes v_{\omega(n)}\right] \longmapsto\left[w_{\sigma \omega(1)} \otimes \cdots \otimes w_{\sigma \omega(n)}\right] \in \bigotimes_{d \in D} W_{d}
$$

with $w_{\sigma \omega(i)}:=\varphi_{\omega(i)}\left(v_{\omega(i)}\right) \in W_{\sigma \omega(i)}, 1 \leq i \leq n$, defines a natural map

$$
\overline{(\sigma, \varphi)}: \bigotimes_{c \in S} V_{c} \rightarrow \bigotimes_{d \in D} W_{d}
$$

of unordered products

Proof. A direct verification.

A particularly important case of the above lemma is when $V_{c}=V_{d}=V$ for all $c \in S$, $d \in D$, and $\varphi_{c}: V \rightarrow V$ is the identity for all $c \in S$. Lemma 4 then gives a natural map

$$
\bar{\sigma}:=\overline{(\sigma, \varphi)}: \bigotimes_{c \in S} V_{c} \rightarrow \bigotimes_{d \in D} V_{d}
$$

Lemma 5. For disjoint finite sets $S^{\prime}, S^{\prime \prime}$, one has a canonical isomorphism

$$
\bigotimes_{c^{\prime} \in S^{\prime}} V_{c^{\prime}} \otimes \bigotimes_{c^{\prime \prime} \in S^{\prime \prime}} V_{c^{\prime \prime}} \cong \bigotimes_{c \in S^{\prime} \sqcup S^{\prime \prime}} V_{c}
$$

Proof. Each $\omega^{\prime}:\{1, \ldots, n\} \stackrel{\cong}{\rightarrow} S^{\prime}$ and $\omega^{\prime \prime}:\{1, \ldots, m\} \stackrel{\cong}{\rightarrow} S^{\prime \prime}$ determine an isomorphism

$$
\omega^{\prime} \sqcup \omega^{\prime \prime}:\{1, \ldots, n+m\} \stackrel{\cong}{\longrightarrow} S^{\prime} \sqcup S^{\prime \prime}
$$

by the formula

$$
\left(\omega^{\prime} \sqcup \omega^{\prime \prime}\right)(i):= \begin{cases}\omega^{\prime}(i), & \text { if } 1 \leq i \leq n, \text { and } \\ \omega^{\prime \prime}(i-n), & \text { if } n<i \leq n+m .\end{cases}
$$

The isomorphism of the lemma is then given by the assignment

$$
\left[v_{\omega^{\prime}(1)} \otimes \cdots \otimes v_{\omega^{\prime}(n)}\right] \otimes\left[v_{\omega^{\prime \prime}(1)} \otimes \cdots \otimes v_{\omega^{\prime \prime}(m)}\right] \longmapsto\left[v_{\left(\omega^{\prime} \sqcup \omega^{\prime \prime}\right)(1)} \otimes \cdots \otimes v_{\left(\omega^{\prime} \sqcup \omega^{\prime \prime}\right)(n+m)}\right] .
$$

This finishes the proof.

The input data of the odd modular endomorphism operad is a graded vector space $V$ with a symmetric tensor $s \in V \otimes V$ of degree +1 . The symmetry means that $\tau(s)=s$, where $\tau$ is the interchange (3). We will interpret as usual $s$ as a linear degree +1 map $s: \mathrm{k} \rightarrow V \otimes V$. For a finite set $S$ put

$$
\mathcal{E}_{n d_{V}}(S):=\underline{\operatorname{Vect}}\left(\bigotimes_{c \in S} V_{c}, \mathrm{k}\right)=\left(\bigotimes_{c \in S} V_{c}\right)^{*}
$$

where $V_{c}:=V$ for each $c \in S$. Given an isomorphism $\sigma: S \rightarrow D$ of finite sets, we define the induced map

$$
\mathcal{E}_{n} d_{V}(\sigma): \mathcal{E} n d_{V}(S) \rightarrow \mathcal{E} n d_{V}(D)
$$

by $\mathcal{E}_{n d_{V}}(\sigma)(x):=x \bar{\sigma}^{-1}$ for $x: \bigotimes_{c \in S} V_{c} \rightarrow \mathrm{k} \in \mathcal{E}_{n d_{V}}(S)$ and $\bar{\sigma}$ as in (11).

Let $S_{1}, S_{2}$ be disjoint finite sets and $a \neq b$ two symbols. Our next task will be to define, for linear functionals

$$
x \in \mathcal{E}_{n} d_{V}\left(S_{1} \sqcup\{a\}\right) \text { and } y \in \mathcal{E} n d_{V}\left(S_{2} \sqcup\{b\}\right),
$$

[odd.tex]

[August 10, 2016] 
their ${ }_{a} \bullet_{b}$-product $x_{a} \bullet_{b} y \in \mathcal{E} n d_{V}\left(S_{1} \sqcup S_{2}\right)$. The natural choice is obviously the composition

$$
\begin{aligned}
& \bigotimes_{c \in S_{1} \sqcup S_{2}} V_{c} \cong \bigotimes_{c^{\prime} \in S_{1}} V_{c^{\prime}} \otimes \bigotimes_{c^{\prime \prime} \in S_{2}} V_{c^{\prime \prime}} \cong \bigotimes_{c^{\prime} \in S_{1}} V_{c^{\prime}} \otimes \mathrm{k} \otimes \bigotimes_{c^{\prime \prime} \in S_{2}} V_{c^{\prime \prime}} \\
& \stackrel{\mathbb{1} \otimes s \otimes \mathbb{1}}{\longrightarrow} \bigotimes_{c^{\prime} \in S_{1}} V_{c^{\prime}} \otimes V_{a} \otimes V_{b} \otimes \bigotimes_{c^{\prime \prime} \in S_{2}} V_{c^{\prime \prime}} \stackrel{\cong}{\longrightarrow} \bigotimes_{c^{\prime} \in S_{1} \sqcup\{a\}} V_{c^{\prime}} \otimes \bigotimes_{c^{\prime \prime} \in S_{2} \sqcup\{b\}} V_{c^{\prime \prime}} \stackrel{x \otimes y}{\longrightarrow \mathrm{k}}
\end{aligned}
$$

in which the isomorphisms are those of Lemma 5. In shorthand,

$$
x_{a} \bullet_{b} y=(x \otimes y)\left(1_{V \otimes S_{1}} \otimes s \otimes 1_{V \otimes S_{2}}\right)
$$

and, denoting $1_{S_{1}}:=1_{V}^{\otimes S_{1}}$ and $1_{S_{2}}:=1_{V}^{\otimes S_{2}}$, still more concisely

$$
x_{a} \bullet_{b} y:=(x \otimes y)\left(1_{S_{1}} \otimes s \otimes 1_{S_{2}}\right) .
$$

Alternatively, one may define $x_{a} \bullet_{b} y$ as the result of the application of the composition

$$
\begin{aligned}
& \left(\bigotimes_{c^{\prime} \in S_{1} \sqcup\{a\}} V_{c^{\prime}}\right)^{*} \otimes\left(\bigotimes_{c^{\prime \prime} \in S_{2} \sqcup\{b\}} V_{c^{\prime \prime}}\right)^{*} \hookrightarrow\left(\bigotimes_{c^{\prime} \in S_{1} \sqcup\{a\}} V_{c^{\prime}} \otimes \bigotimes_{c^{\prime \prime} \in S_{2} \sqcup\{b\}} V_{c^{\prime \prime}}\right)^{*} \\
& \quad \stackrel{\cong}{\longrightarrow}\left(\bigotimes_{c^{\prime} \in S_{1}} V_{c^{\prime}} \otimes V_{a} \otimes V_{b} \otimes \bigotimes_{c^{\prime \prime} \in S_{2}} V_{c^{\prime \prime}}\right)^{*} \stackrel{(\mathbb{1} \otimes s \otimes \mathbb{1})^{*}}{\longrightarrow}\left(\bigotimes_{c^{\prime} \in S_{1}} V_{c^{\prime}} \otimes \mathrm{k} \otimes \bigotimes_{c^{\prime \prime} \in S_{2}} V_{c^{\prime \prime}}\right)^{*} \\
& \quad \cong\left(\bigotimes_{c^{\prime} \in S_{1}} V_{c^{\prime}} \otimes \bigotimes_{c^{\prime \prime} \in S_{2}} V_{c^{\prime \prime}}\right)^{*} \stackrel{\cong}{\longrightarrow}\left(\bigotimes_{c \in S_{1} \sqcup S_{2}} V_{c}\right)^{*}
\end{aligned}
$$

to $x \otimes y \in\left(\otimes_{c^{\prime} \in S_{1} \sqcup\{a\}} V_{c^{\prime}}\right)^{*} \otimes\left(\otimes_{c^{\prime \prime} \in S_{2} \sqcup\{b\}} V_{c^{\prime \prime}}\right)^{*}$. In shorthand,

$$
x_{a} \bullet b:=\left(1_{S_{1}} \otimes s \otimes 1_{S_{2}}\right)^{*}(x \otimes y) .
$$

We shall keep in mind that (12b) implicitly involves canonical identifications and inclusions.

An obvious way to define the contraction $\bullet_{u v} x \in \mathcal{E} n d_{V}(S ; g+1)$ of a linear functional $x \in \mathcal{E}_{n} d_{V}(S \sqcup\{u, v\} ; g)$ is the composition

$$
\bigotimes_{c \in S} V_{c} \cong \mathrm{k} \otimes \bigotimes_{c \in S} V_{c} \stackrel{s \otimes \mathbb{1}}{\longrightarrow} V_{u} \otimes V_{v} \otimes \bigotimes_{c \in S} V_{c} \cong \bigotimes_{c \in S \sqcup\{u, v\}} V_{c} \stackrel{x}{\longrightarrow} \mathrm{k} .
$$

In shorthand,

$$
\bullet \text { uv } x:=x\left(s \otimes 1_{S}\right) .
$$

The commutativity of the diagram

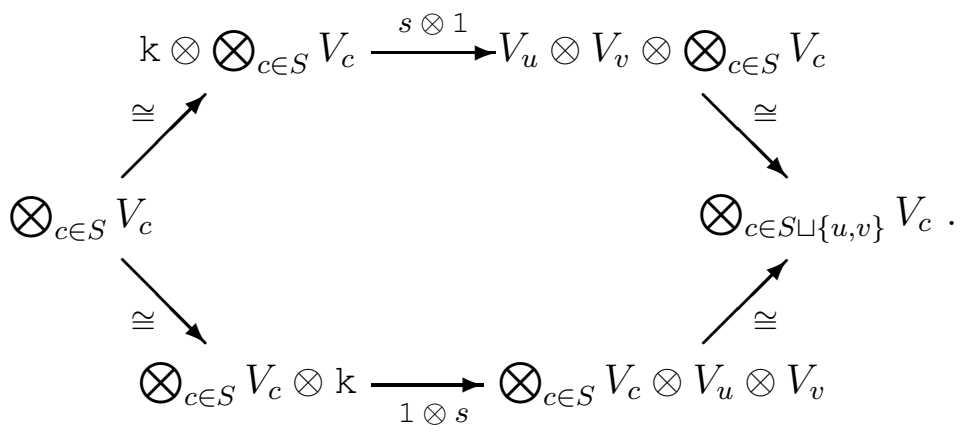

implies that we could replace $\left(s \otimes 1_{S}\right)$ in (13a) by $\left(1_{S} \otimes s\right)$ with the same result. We could in fact place $s$ into an arbitrary position without affecting the result. A similar remark applies also to (12a) and (12b). 
Another possibility is to define $\bullet_{u v} x$ as the result of the application

$$
\left(\bigotimes_{c \in S \sqcup\{u, v\}} V_{c}\right)^{*} \cong\left(V_{u} \otimes V_{v} \otimes \bigotimes_{c \in S} V_{c}\right)^{*} \stackrel{(s \otimes \mathbb{1})^{*}}{\longrightarrow}\left(\mathrm{k} \otimes \bigotimes_{c \in S} V_{c}\right)^{*} \cong\left(\bigotimes_{c \in S} V_{c}\right)^{*}
$$

to $x \in\left(\bigotimes_{c \in S \sqcup\{u, v\}} V_{c}\right)^{*}$. In shorthand,

$$
\bullet_{u v} x:=\left(s \otimes 1_{S}\right)^{*}(x) .
$$

Here comes a surprise. Since $|s|=1$, the two definitions of the ${ }_{a} \bullet_{b}$-operation, i.e. the one via (12a) and the one via (12b), lead to different results! The reason is that they are not dual to each other, since the duality (2) acquires a nontrivial sign. The resulting $x_{a} \bullet_{b} y$ 's differ by $(-1)^{|x|+|y|}$.

Likewise, definitions $(13 \mathrm{a})$ and $(13 \mathrm{~b})$ are not dual to each other and the resulting $\bullet_{u v}(x)$ 's differ by $(-1)^{|x|}$. What happens is described in the following proposition; recall that Vect and $\underline{\text { Vect }}_{\mathrm{M}}$ denote the two versions of the category Vect discussed in Section 11.

Proposition 6. The modular collection $\mathcal{E}_{n} d_{V}$ with operations ${ }_{a} \bullet_{b}$ and $\bullet_{u v}$ defined by (120) and (13b) is an odd modular operad in $\underline{\text { Vect }}_{\mathrm{S}}$ while (12a) and (13a) give an odd modular operad in $\underline{\text { Vect }}_{\mathrm{M}}$.

Proof. Let us show that the ${ }_{a} \bullet_{b}$-operations defined by (12b) satisfy (7a). In the following calculations, $\bar{s}$ and $\overline{\bar{s}}$ are two copies of the map $s: \mathrm{k} \rightarrow V \otimes V$. One has

$$
\begin{aligned}
x_{a} \bullet_{b}\left(y_{c} \bullet_{d} z\right) & =\left(1_{S_{1}} \otimes \bar{s} \otimes 1_{S_{2} \sqcup S_{3}}\right)^{*}\left(x \otimes\left(y_{c} \bullet_{d} z\right)\right) \\
& =\left(1_{S_{1}} \otimes \bar{s} \otimes 1_{S_{2} \sqcup S_{3}}\right)^{*}\left(x \otimes\left(1_{S_{2} \sqcup\{b\}} \otimes \overline{\bar{s}} \otimes 1_{S_{3}}\right)^{*}(y \otimes z)\right) \\
& =(-1)^{|x|}\left(1_{S_{1}} \otimes \bar{s} \otimes 1_{S_{2} \sqcup S_{3}}\right)^{*}\left(1_{S_{1} \sqcup\{a, b\} \sqcup S_{2}} \otimes \overline{\bar{s}} \otimes 1_{S_{3}}\right)^{*}(x \otimes y \otimes z)
\end{aligned}
$$

while

$$
\begin{aligned}
\left(x_{a} \bullet_{b} y\right)_{c} \bullet_{d} z & =\left(1_{S_{1} \sqcup S_{2}} \otimes \overline{\bar{s}} \otimes 1_{S_{3}}\right)^{*}\left(\left(x_{a} \bullet_{b} y\right) \otimes z\right) \\
& =\left(1_{S_{1} \sqcup S_{2}} \otimes \overline{\bar{s}} \otimes 1_{S_{3}}\right)^{*}\left(\left(1_{S_{1}} \otimes \bar{s} \otimes 1_{S_{2} \sqcup\{c\}}\right)^{*}(x \otimes y) \otimes z\right) \\
& =\left(1_{S_{1} \sqcup S_{2}} \otimes \overline{\bar{s}} \otimes 1_{S_{3}}\right)^{*}\left(1_{S_{1}} \otimes \bar{s} \otimes 1_{S_{2} \sqcup\{c, d\} \sqcup S_{3}}\right)^{*}(x \otimes y \otimes z) .
\end{aligned}
$$

To finish the proof of $(\overline{\nabla a})$, we observe that

$$
\begin{aligned}
\left(1_{S_{1}} \otimes \bar{s} \otimes 1_{S_{2}} \otimes \overline{\bar{s}} \otimes 1_{S_{3}}\right)^{*} & =\left(1_{S_{1}} \otimes \bar{s} \otimes 1_{S_{2} \sqcup S_{3}}\right)^{*}\left(1_{S_{1} \sqcup\{a, b\} \sqcup S_{2}} \otimes \overline{\bar{s}} \otimes 1_{S_{3}}\right)^{*} \\
& =-\left(1_{S_{1} \sqcup S_{2}} \otimes \overline{\bar{s}} \otimes 1_{S_{3}}\right)^{*}\left(1_{S_{1}} \otimes \bar{s} \otimes 1_{S_{2} \sqcup\{c, d\} \sqcup S_{3}}\right)^{*},
\end{aligned}
$$

the minus sign coming from commuting $\bar{s}$ over $\overline{\bar{s}}$.

Let us also verify explicitly that the ${ }_{a} \bullet_{b}$-operations defined by (12a) satisfy (7b). The related calculation is of course obtained from the above one by removing duals and inverting the order of compositions but, very crucially, without inserting Koszul signs. We obtain

$$
\begin{aligned}
x_{a} \bullet_{b}\left(y_{c} \bullet_{d} z\right) & =\left(x \otimes\left(y_{c} \bullet_{d} z\right)\right)\left(1_{S_{1}} \otimes \bar{s} \otimes 1_{S_{2} \sqcup S_{3}}\right) \\
& =\left(x \otimes(y \otimes z)\left(1_{S_{2} \sqcup\{b\}} \otimes \overline{\bar{s}} \otimes 1_{S_{3}}\right)\right)\left(1_{S_{1}} \otimes \bar{s} \otimes 1_{S_{2} \sqcup S_{3}}\right) \\
& =(x \otimes y \otimes z)\left(1_{S_{1} \sqcup\{a, b\} \sqcup S_{2}} \otimes \overline{\bar{s}} \otimes 1_{S_{3}}\right)\left(1_{S_{1}} \otimes \bar{s} \otimes 1_{S_{2} \sqcup S_{3}}\right)
\end{aligned}
$$

on one hand and

$$
\left(x_{a} \bullet_{b} y\right)_{c} \bullet_{d} z=\left(\left(x_{a} \bullet_{b} y\right) \otimes z\right)\left(1_{S_{1} \sqcup S_{2}} \otimes \overline{\bar{s}} \otimes 1_{S_{3}}\right)
$$

[odd.tex]

[August 10, 2016] 


$$
\begin{aligned}
& =\left((x \otimes y)\left(1_{S_{1}} \otimes \bar{s} \otimes 1_{S_{2} \sqcup\{c\}}\right) \otimes z\right)\left(1_{S_{1} \sqcup S_{2}} \otimes \overline{\bar{s}} \otimes 1_{S_{3}}\right) \\
& =(-1)^{|z|}(x \otimes y \otimes z)\left(1_{S_{1}} \otimes \bar{s} \otimes 1_{S_{2} \sqcup\{c, d\} \sqcup S_{3}}\right)\left(1_{S_{1} \sqcup S_{2}} \otimes \overline{\bar{s}} \otimes 1_{S_{3}}\right)
\end{aligned}
$$

on the other. Axiom (7b) now follows from the equality

$$
\begin{aligned}
\left(1_{S_{1}} \otimes \bar{s} \otimes 1_{S_{2}} \otimes \overline{\bar{s}} \otimes 1_{S_{3}}\right) & =\left(1_{S_{1}} \otimes \bar{s} \otimes 1_{S_{2} \sqcup\{c, d\} \sqcup S_{3}}\right)\left(1_{S_{1} \sqcup S_{2}} \otimes \overline{\bar{s}} \otimes 1_{S_{3}}\right) \\
& =-\left(1_{S_{1} \sqcup\{a, b\} \sqcup S_{2}} \otimes \overline{\bar{s}} \otimes 1_{S_{3}}\right)\left(1_{S_{1}} \otimes \bar{s} \otimes 1_{S_{2} \sqcup S_{3}}\right) .
\end{aligned}
$$

Notice that the sign difference between the results of the above two computations is $(-1)^{|x|}$ versus $(-1)^{|z|}$ as it should be. The verification of axioms (8a) resp. (8b) is similar. The remaining axioms are not affected by the choice of the monoidal structure in Vect so we will not verify them here.

We evaluated (12a), (12b), (13a) and (13b) inside the standard monoidal structure of Vect. If we use the McGill one, then (12a) and (13a) would give an odd modular operad in Vect ${ }_{\mathrm{S}}$ while (12b) and (13b) would lead to an odd modular operad in Vect ${ }_{M}$.

\section{REFERENCES}

[1] M. Doubek, B. Jurčo, and K. Muenster. Modular operads and the quantum open-closed homotopy algebra. Preprint arXiv:1308.3223.

[2] E. Getzler and M.M. Kapranov. Modular operads. Compos. Math., 110(1):65-126, 1998.

[3] R.M. Kaufmann, B.C. Ward and J.J. Zuniga. The odd origin of Gerstenhaber brackets, Batalin-Vilkovisky operators and the master equations. Journal of Math. Phys., 56:103504(40 pages), 2015.

[4] M. Markl. Loop homotopy algebras in closed string field theory. Comm. Math. Phys., 221(2):367-384, 2001.

[5] M. Markl, S. Shnider, and J.D. Stasheff. Operads in algebra, topology and physics, volume 96 of Mathematical Surveys and Monographs. American Mathematical Society, Providence, RI, 2002.

[6] C. Muenster and I. Sachs. On Homotopy Algebras and Quantum String Field Theory. Miscolc Math. Notes, 14(2):433-443, 2013.

[7] K. Münster and I. Sachs. Quantum open-closed homotopy algebra and string field theory. Comm. Math. Phys., 321(3):769-801, 2013.

Mathematical Institute of the Academy, Žitná 25, 11567 Prague 1, The Czech Republic

Faculty of Mathematics and Physics, Charles University, 18675 Sokolovská 83, Prague 8, The Czech Republic

E-mail address: markl@math.cas.cz

[August 10, 2016]

[odd.tex] 